\title{
Selective Laser Sintering of Calcium Polyphosphate: Finite Element Modeling and Experiments
}

\author{
Yaser Shanjani, Ehsan Toyserkani
Rapid Prototyping Laboratory, Department of Mechanical and Mechatronics Engineering, University of Waterloo, Waterloo, Ontario, Canada
E-mail: yshanjan@uwaterloo.ca

\begin{abstract}
In this paper, the selective laser sintering (SLS) process of a bio-composite blend comprising of biodegradable calcium polyphosphate (CPP) and polyvinyl alcohol (PVA), as a polymeric binder, is investigated. A multi-physics finite element model including both thermal and viscous sintering phenomena was developed and simulated in different process conditions. The model was used to predict temperature and porosity distribution of sintered regions throughout the process domain and period. It is observed that the porosity of the sintered region is a logistic function of the laser scanning speed and a negative logistic function of the laser power. The thickness of the fabricated CPP layers, using an experimental setup, fairly demonstrates an agreement with the simulation.

DOI: $10.2961 / \mathrm{jlmn} .2009 .01 .0006$
\end{abstract}

Keywords: selective laser sintering, liquid phase sintering, calcium polyphosphate, porosity, heat affected zone

\section{Introduction}

Selective laser sintering (SLS) enables the manufacturing of parts from powdered material by selectively heating and fusing of powder particles using a laser beam [1]. To realize the fabrication of complexshaped implants/scaffolds composed of calcium polyphosphate (CPP), which is a bio-degradable ceramic with polymeric chains [2], by the SLS technique, an admixture of CPP and a polymeric binder or a polymeric coated CPP powder is used. The powder is spread as sequential layers on the former ones. Then, the laser beam rasters the powder bed and acts as incoming concentrated thermal energy. The low melting-temperature polymer flows and merges when it is heated by the laser beam and resolidifies when the laser beam passes. Consequently, the polymer acts as a binder and binds the CPP particles together. In other words, primary binding is conducted in the selected segments of the powder bed via viscous sintering of the polymeric component. The particles within the heat affected zone (HAZ) are bonded to the beneath layers as well. The process repeats until the desired green object completes. In the final step, in order to increase the strength of the model, the green part is heat treated in a furnace to decompose the binder followed by sintering process. Fig. 1 and Fig. 2 show the schematic of liquid phase selective laser sintering process and its mechanism.

The strength and the density of prototyped objects as well as their geometrical features are very dependent on the particle binding during the laser sintering stage. Therefore, for the optimization of the prototyping process, the prediction of thermal behaviour of the powder bed under the moving laser heat source through a numerical modeling is of importance.

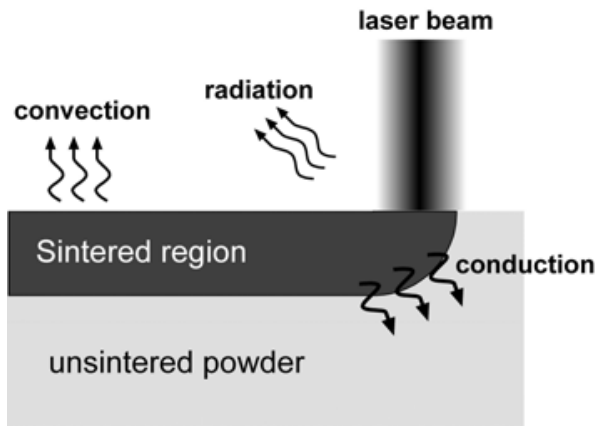

Fig. 1 Schematic of selective laser sintering of powder

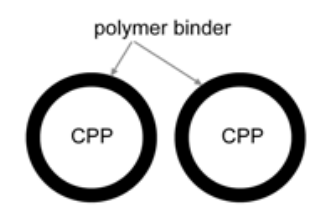

(a)

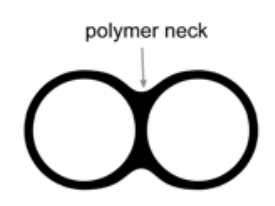

(b)
Fig. 2 Schematic of CPP liquid phase sintering; (a) before and (b) after laser sintering

Several studies on the modeling of the SLS process have been performed. Thermal models of SLS in onedimensional $[3,4]$, two-dimensional $[5,6]$, and threedimensional forms [7] have been reported. Nelson et al. constructed a thermal model of the SLS process for predicting the layer-to-layer fusion of polycarbonate powder [3]. This model can predict, to within a $20 \%$ accuracy, the effects of the primary process variables such as the scan speed, scan overlap, and laser power on the fusion depths of the polycarbonate (PC) powder. Moreover, Nelson's finite difference model estimates the sintering depths in poly(methyl methacrylate) (PMMA) and coated silicon carbide (SiC) powders [4]. The SLS of an amorphous polymer, as well as a crystalline and glass-filled polymer was simulated by Childs et al. [6], using a 
sequential thermal finite element method. A numerical simulation of two-dimensional melting and resolidification of a two-component metal powder layer in the SLS process was developed by Chen et al. [8]. The numerical prediction of the temperature and density distribution in SLS process, based on Mackenzie and Shuttelworth's model of the sintering rate was published by Cervera et al. [7]. He has eliminated the time parameter by using a local coordinate system, located at the centre of the moving laser beam. Recently, Dong et al. [9] developed a model in ABAQUS with a bi-level structure integration procedure. In his model, the density and the heat equation are integrated at the outer and inner levels, respectively.

This work addresses the analysis of selective laser sintering of CPP, which is highly receiving attentions as a suitable candidate for osteochondral implants [10-11], through a "multi-physics" finite element model. Since the porosity of an osteochondral scaffold has a great effect on the bio-mechanical properties, the characterization of SLS process may provide a control on the macro-porosity of the CPP scaffolds.

\section{Heat transfer analysis in the SLS process}

In the binding stage of the SLS process, the heat transfer behaviour in the substrate is described by the classical heat conduction equation:

$$
\rho \cdot C p \cdot \frac{\partial T}{\partial t}=\nabla(k \cdot \nabla T)+Q_{g e n}
$$

where $\rho$ is the density, $C p$ is the specific heat, $T$ is the temperature, $t$ is the time, $k$ is the thermal conductivity, and $Q_{\text {gen }}$ represents the heat generated in the physical body.

On the boundaries, Equation (2) is applicable:

$$
n \cdot q=Q_{\text {Laser }}-h_{\text {conv }} \cdot\left(T-T_{\infty}\right)-\varepsilon_{\text {ems }} \cdot \sigma \cdot\left(T^{4}-T_{\text {surr }}^{4}\right)
$$

where $n$ is the normal vector of the heat flux plan, $q$ is the heat flux, $h_{\text {conv }}$ is the thermal convection coefficient, $\varepsilon_{e m s}$ is the emissivity, $\sigma$ is the Stefan-Boltzmann constant $\left(5.67 \mathrm{E}-8 \mathrm{~W} / \mathrm{m}^{2} \cdot{ }^{\circ} \mathrm{K}^{4}\right)$.

It is obvious that $Q_{\text {Laser }}$ is applied only on the top surface of the powder bed. If $h_{\text {conv }}$ is considered for a combined radiation and convection heat transfer [12], the boundary condition is simplified as

$$
n \cdot q=Q_{\text {Laser }}-h_{\text {conv }} \cdot\left(T-T_{\infty}\right)
$$

\subsection{Laser irradiation submodel}

To determine the concentrated heat flux of the laser beam on the top surface of the substrate, a characterization of the beam and energy profile is required. By assuming a Gaussian laser beam with $\mathrm{TEM}_{00}$ mode, the laser beam intensity profile is

$$
I(r)=I_{0} \cdot \exp \left(-\frac{r^{2}}{a^{2}}\right)
$$

where $r$ is the radial component in a polar coordination system, and $I_{0}$ is the intensity scale factor that is defined by the laser power and the characteristic radius of the laser intensity profile, $a$, as [13]

$$
\begin{aligned}
& I_{0}=\frac{\beta \cdot P}{\pi \cdot a^{2} \cdot\left[1-\exp \left(-\frac{r_{\text {beam }}^{2}}{a^{2}}\right)\right]} \\
& a=\frac{\sqrt{2}}{2} r_{\text {beam }}
\end{aligned}
$$

where $\beta$ is the absoptivity and $P$ is the laser power.

In Cartesian coordinate system, the laser incident radius is as follows:

$$
r=\sqrt{\left(x-x_{0}\right)^{2}+\left(y-y_{0}\right)^{2}}
$$

Consequently, the moving laser is defined as

$$
\begin{aligned}
& Q_{\text {Laser }}=I_{0} \cdot e^{\left(-\frac{\left(x-v_{x} \cdot t-x_{0}\right)^{2}+\left(y-v_{y} \cdot t-y_{0}\right)^{2}}{a^{2}}\right)} \times \\
& {\left[\left(\left(x-v_{x} \cdot t-x_{0}\right)^{2}+\left(y-v_{y} \cdot t-y_{0}\right)^{2}\right)<r_{\text {beam }}{ }^{2}\right]}
\end{aligned}
$$

where $v_{x}$ and $v_{y}$ are the laser moving velocity components in $x$ and $y$ directions, respectively. The last term of (7) identifies the moving of the heat flux.

\subsection{Powder bed material properties submodel}

The density ( $\left.\rho_{\text {composite }}\right)$ of the composite powder, consisting CPP and the polymeric binder, is calculated from the densities of the components as follows:

$$
\rho_{\text {composite }}=\phi_{\text {polymer }} \cdot \rho_{\text {polymer }}+\left(1-\phi_{\text {polymer }}\right) \cdot \rho_{\text {ceramic }}
$$

where $\phi$ is the volume fraction of the components.

The specific heat of the composite powder $C p$ is estimated from the mass fraction ( $\omega$ ) average of the specific heats of the polymer and the ceramic by the calculation of

$$
\begin{aligned}
& C p=\omega_{\text {polymer }} \cdot C p_{\text {polymer }}+\left(1-\omega_{\text {polymer }}\right) \cdot C p_{\text {ceramic }} \\
& \omega_{\text {polymer }}=\frac{\phi_{\text {polymer }} \cdot \rho_{\text {polymer }}}{\phi_{\text {polymer }} \cdot \rho_{\text {polymer }}+\left(1-\phi_{\text {polymer }}\right) \cdot \rho_{\text {ceramic }}}
\end{aligned}
$$

Yagi et al. [14] also formulated a more accurate model for the effective conductivity of a packed bed, in which the conduction, convection and radiation effects within the powder bed are considered. The model has been developed for low-temperature and high-temperature conditions. At low-temperatures, only convection causes the heat conductivity, whereas radiation evidently enhances the heat transfer by [9]

Low Temperature: 


$$
k=\frac{(1-\varepsilon) \cdot k_{\text {solid }}(T)}{1+\psi_{k} \cdot \frac{k_{\text {solid }}(T)}{k_{\text {gas }}}}
$$

High Temperature:

$$
\begin{gathered}
k=\frac{\beta \cdot(1-\varepsilon)}{\frac{\gamma}{k_{\text {solid }}(T)}+\frac{1}{\frac{k_{\text {gas }}}{\psi}+D_{p} \cdot h_{\mathrm{rs}}}}+\varepsilon \cdot \beta_{k} \cdot D_{p} \\
\varepsilon=\left(\rho_{\text {solid }}-\rho_{\text {composite }}\right) / \rho_{\text {solid }}
\end{gathered}
$$

where $k_{\text {solid }}$ is the conductivity of the powder material, $k_{\text {gas }}$ is the conductivity of air, $\varepsilon$ is the void fraction (porosity) of the bed, and $\psi$ is an empirical coefficient normally considered as [9]

$\psi=0.193 \cdot \varepsilon^{1.854}$

$k_{\text {solid }}$ is derived for a solid composite particle which includes a ceramic core with the overall radius, $R_{c}$, and a polymeric coating with the thickness, $t_{t h}$, by [2]

$$
\begin{aligned}
& \frac{1}{k_{\text {solid }}}=\left(\frac{B}{k_{\text {ceramic }}^{2}}-\frac{2}{k_{\text {ceramic }}}\right) \cdot \ln \left(\frac{B-k_{\text {ceramic }}}{B-k_{\text {ceramic }} \cdot\left(\frac{t_{\text {th }}}{R_{c}}\right)}\right)+\frac{\left(\frac{t_{\text {th }}}{R_{c}}\right)}{k_{\text {polymer }}}+\left(\frac{1-\left(\frac{t_{\text {th }}}{R_{c}}\right)}{k_{\text {ceramic }}}\right) \\
& B=2 \cdot\left(\frac{t_{\text {th }}}{R_{c}}\right) \cdot k_{\text {polymer }}+2 \cdot\left(1-\left(\frac{t_{\text {th }}}{R_{c}}\right)\right) \cdot k_{\text {ceramic }}
\end{aligned}
$$

\subsection{Sintering submodel}

The modelling of SLS requires simultaneous solution of equations for the rate of the porosity collapse and the changes in local thermal properties. The change in the porosity occurs due to the viscous sintering and merging of the polymeric constituent which causes the CPP particles to rearrange and be closer to each other. The rate of porosity collapse is described as [2]

$$
-d \varepsilon / d t=k^{\prime} \cdot\left(\varepsilon-\varepsilon_{\infty}\right)
$$

where $\varepsilon$ is the porosity of powder bed and $\varepsilon_{\infty}$ is the plateau porosity at sintering temperature, $T$, which is assumed to be zero. Since sintering is a rate process, it is reasonable to assume that the rate constant $\left(k^{\prime}\right)$ follows an Arrhenius form:

$$
k^{\prime}=A \cdot \exp \left(-E_{a} / R_{G a s} T\right)
$$

where $E_{a}$ is the activation energy, $R_{G a s}$ is the universal Gas constant, and $A$ is a constant. The activation energy of the viscous flow can also be used for sintering $[1,2]$.

\section{Modeling results and discussion}

For this study, polyvinyl alcohol (PVA) was selected as the polymeric binder which was used to be either mixed with the CPP powder or coated on the CPP particles.

Since the viscous flow occurs in the polymeric constituent, the sintering rate data only for PVA is required. The correlation of PVA's porosity over different temperatures and sintering time is plotted in Fig. 3, by using (15) and (16). The initial porosity is assumed to be $54 \%$, based on the reported data of commercial SLS systems [4].

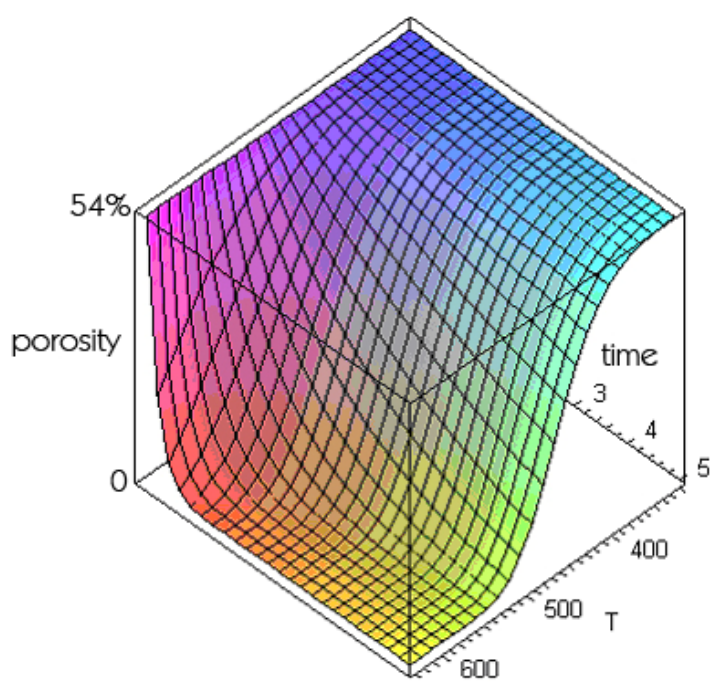

Fig. 3 PVA porosity variation versus time and temperature

For the analysis, a 2D multi-physics finite element (FE) model of the coupled heat transfer and viscous sintering physics was developed. The model is for sintering of a single layer of powder. The COMSOL Multiphysics package was used for this purpose. The simulation parameters are lists in Table 1. The governing equation of sintering was modeled as a time dependent partial differential equation (PDE). The thermal and sintering equations are linked by an expression which relates the thermal conductivity at each point to the powder bed density according to (10) and (11). The strategy for the solution of this set of coupled equations is to solve the PDE and the thermal equation instantaneously for every time step. The conductivity of each node is calculated based on the updated porosity. It means that both physics are updating the required data for each other during the solution. The mesh independency analysis was conducted and the optimum results were obtained with 32000 quadrilateral elements.

Table 1 Simulation parameters

\begin{tabular}{cc}
\hline Parameter & Value \\
\hline PVA vol\% & $20 \%$ \\
Initial porosity & $54 \%$ \\
Laser power & $1.8 \mathrm{~W}$ \\
Laser scanning speed & $5 \mathrm{~mm} / \mathrm{s}$ \\
Preheating temperature & $55^{\circ} \mathrm{C}$ \\
\hline
\end{tabular}


Since the heat affected zone (HAZ) is dramatically small comparing to the whole powder bed, the powder bed was modeled as a rectangular of $20 \times 4 \mathrm{~mm}$. In order to have a reliable and precise solution the mesh at the top surface is finer than the mesh within the other regions. Thus, the Gaussian pattern of the energy is distributed over a number of nodes. Furthermore, since the temperature gradient is quite large in this region, finer mesh will result in a more precise calculation of the temperature distribution. The simulation was performed for several conditions to investigate the effects of the process parameters such as laser power and laser scanning speed on the porosity of sintered portion of the powder.

The temperature distributions on the top surface of powder bed are plotted for any 0.2 seconds in Fig. 4 . The diagram represents an overview of the temperature history on the top surface which is traveled by the laser beam. It is shown that the temperature of the top surface remains about $400^{\circ} \mathrm{K}$ in average, after 1 second.

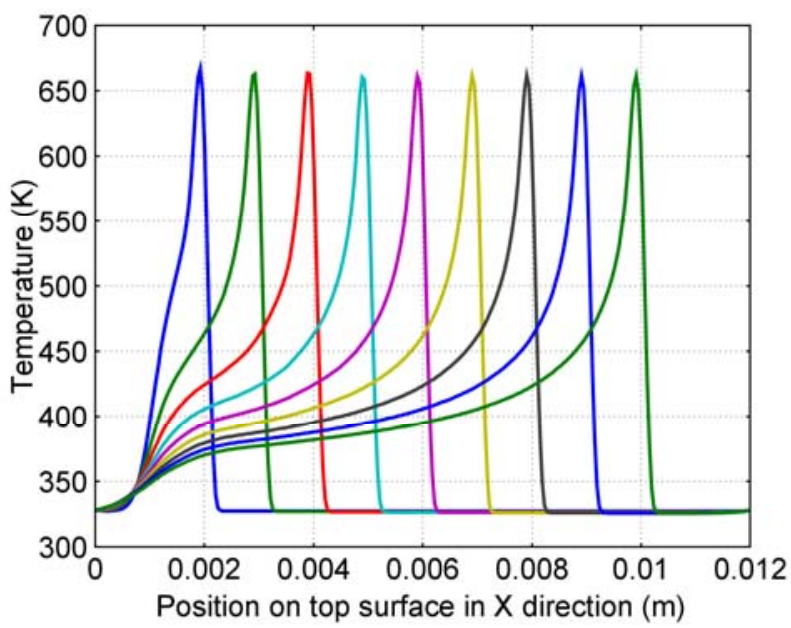

Fig. 4 Temperature on top surface for every $0.2 \mathrm{sec}$

The history of the porosity (void fraction) on the top surface of the powder bed is presented in Fig. 5 for every $0.2 \mathrm{sec}$. A decrease in the porosity, which means an increase in the solidification, even after the laser beam has passed, is obvious. This phenomenon indicates the significant effect of the time in the physics of sintering. It means that the densification of the sintered region continues, with a rate of $k^{\prime}$, even after the laser beam is far from the targeted area.

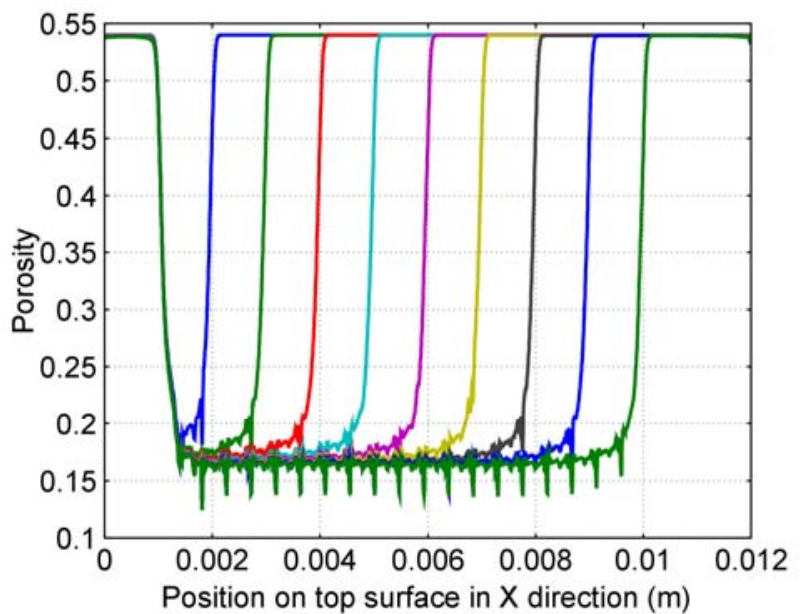

Fig. 5 Porosity on top surface for every $0.2 \mathrm{sec}$

The sintering depth of the powder bed can be determined using the data presented in Fig. 6. It is observed that about $300 \mu \mathrm{m}$ of powder is sintered in the simulation condition. This sintering depth is desired considering that the thickness of the spread powder layer in layer manufacturing is about $125 \mu \mathrm{m}$. It means that the layer sintering condition is appropriate for fabrication of multilayered parts with layers of such thickness. The figure also represents that the porosity of the sintered region decreased about $72 \%$ on the top surface.

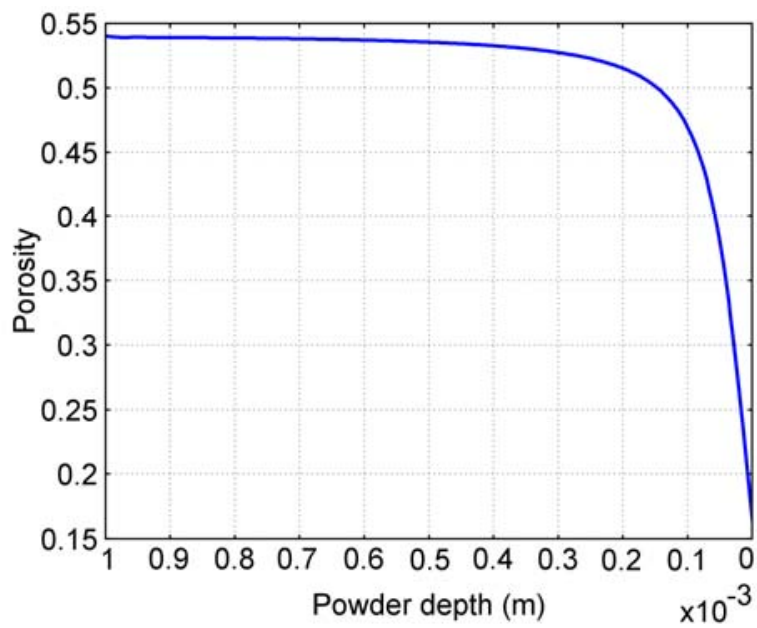

Fig. 6 Porosity variation in powder bed depth

Fig. 7 and Fig. 8, respectively, illustrate the maximum temperature and porosity in the powder bed with different laser power for laser scanning speed of $5 \mathrm{~mm} / \mathrm{s}$. A large decrease in porosity is observed when the power is higher than $1.5 \mathrm{~W}$. Also, it is observed that the porosity does not change remarkably with the laser powers less than $0.5 \mathrm{~W}$. A typical logistic function, as shown in the figure, is fitted on the data. 


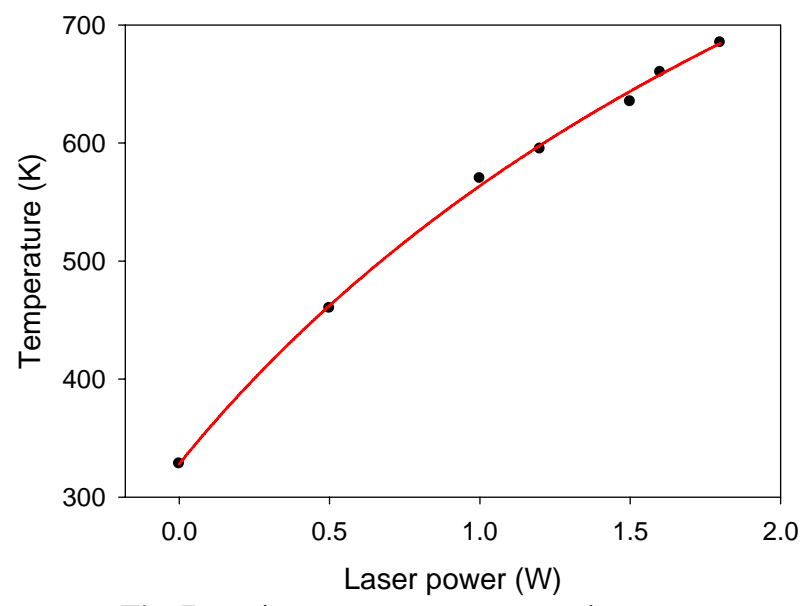

Fig. 7 Maximum temperature versus laser power

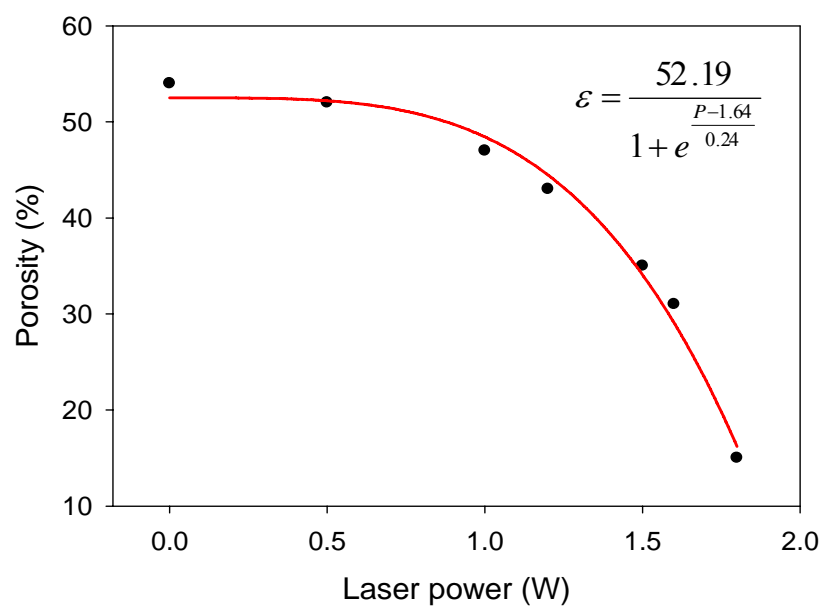

Fig. 8 Minimum porosity versus laser power

Changing the laser scanning speed also influences on the porosity of the sample, as shown in Fig. 9, fixing the laser power to $1.2 \mathrm{~W}$. It is evident that the higher the laser speed, the less the densification. It is noteworthy that the porosity does not change remarkably with the laser scanning speed higher than $7 \mathrm{~mm} / \mathrm{s}$.

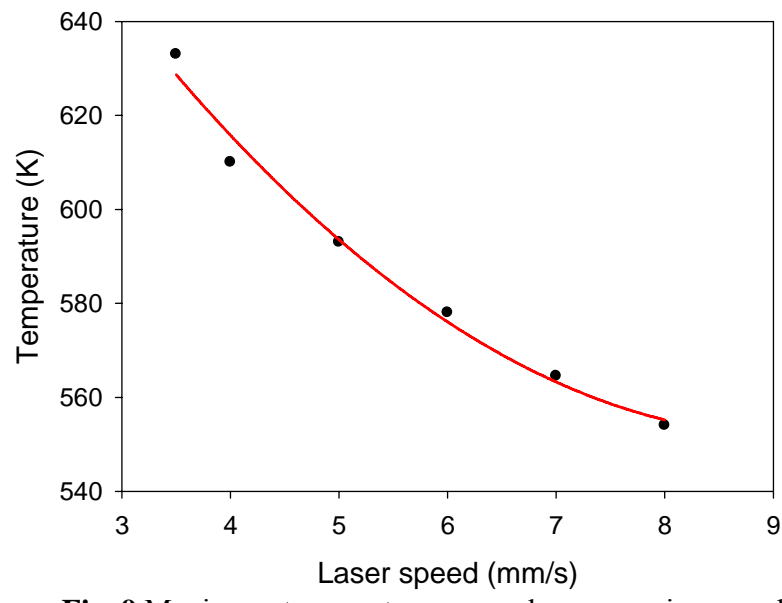

Fig. 9 Maximum temperature versus laser scanning speed

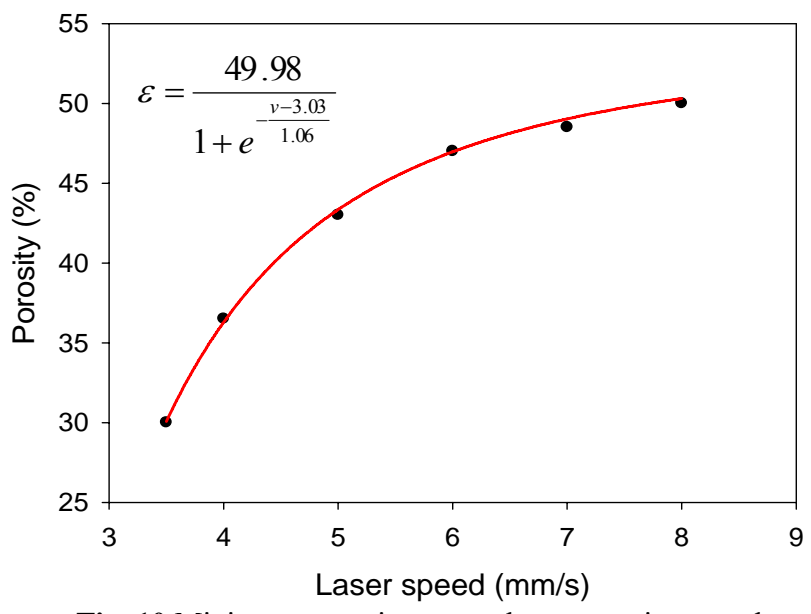

Fig. 10 Minimum porosity versus laser scanning speed

\section{Experiments}

For the feasibility study of the laser sintering of CPP, mixtures of CPP and PVA powders with different volumeratios were prepared. The CPP powder mesh size was 45$75 \mu \mathrm{m}$, whereas the particle size of the PVA powder was less than $63 \mu \mathrm{m}$. The material specifications of the used PVA are listed in Table 2. A CW fibre Erbium laser (IPG Photonics, MA, USA) with a transverse mode of $\mathrm{TEM}_{00}$ and a wavelength of $1550 \mathrm{~nm}$ was utilized for the process. The experimental setup, developed at the University of Waterloo, is shown in Fig. 11. Fig. 12 shows a fabricated layer with the process parameters of $1.8 \mathrm{~W}$ laser power and $5 \mathrm{~mm} / \mathrm{s}$ scanning speed. $20 \mathrm{vol} \%$ PVA was used.

Table 2 Specifications of the used PVA

\begin{tabular}{ccc}
\hline PVA & Hydrolyzed & Molecular Weight \\
\hline$\# 1$ & $98-99 \%$ & Medium \\
$\# 2$ & $86-89 \%$ & Low \\
\hline
\end{tabular}

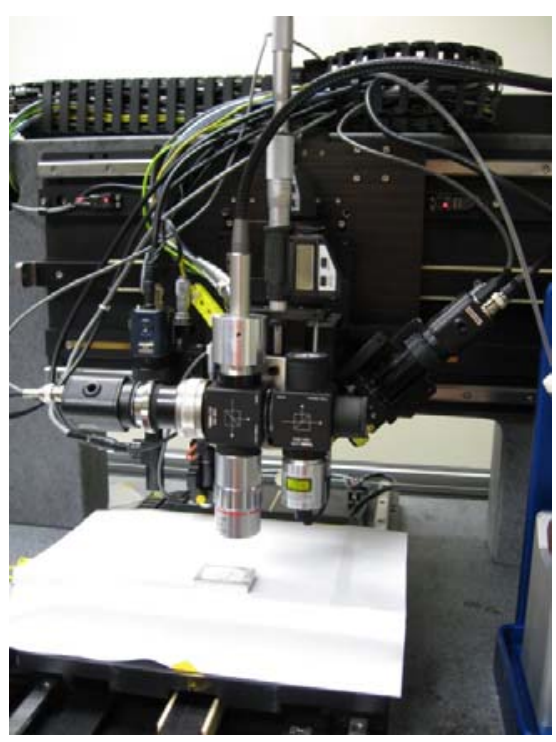

Fig. 11 Experimental setup for laser sintering of CPP 
The section view of the fabricated layer is shown in Fig. 13. To create this sample, the fabricated CPP layer was mounted in an epoxy holder. The thickness of the sample was measured to be approximately $400 \mu \mathrm{m}$ as shown in the figure, whereas the counterpart modeling result was obtained to be $300 \mu \mathrm{m}$. The observed difference between the experimental and modeling results in terms of the layer thickness may be resulted from the transmission of the laser beam through the surface pores and also uncertain laser beam scattering due to non-uniform surface particle distribution (i.e., diffuse reflection explained by Drude reflectivity principle). This phenomenon was not considered in the finite element modeling of the process that may be the source of the discrepancies between the experimental and modeling results.

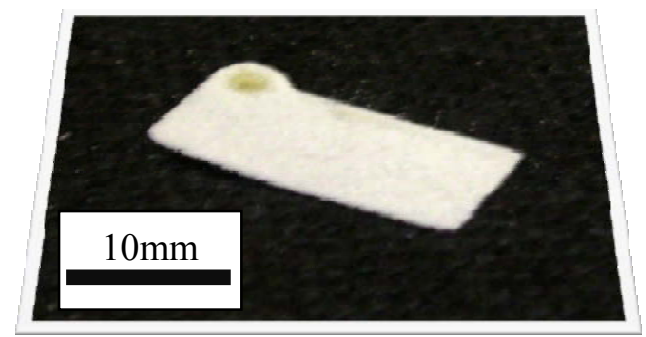

Fig. 12 A thin layer of laser sintered CPP

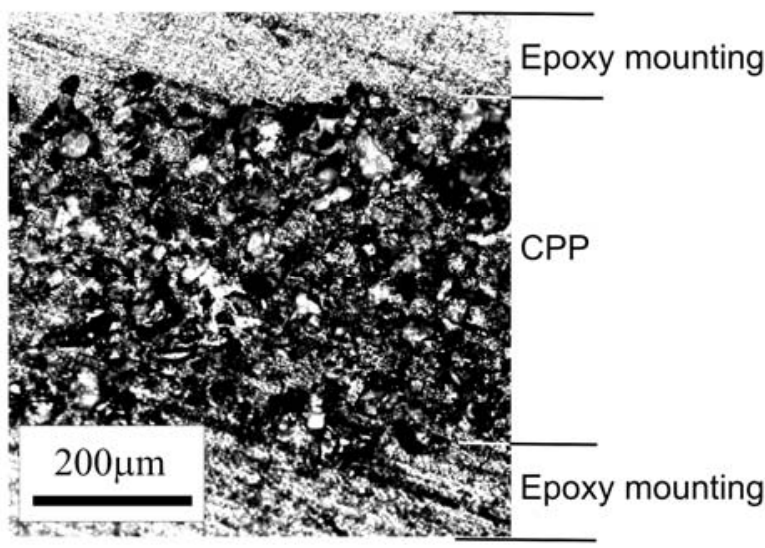

Fig. 13 A microscopic view of the section of a sintered layer

Fig. 14 displays the SEM pictures of two samples which are fabricated with the same process parameters $(16 \mathrm{~W}$ and $45 \mathrm{~mm} / \mathrm{s}$ ) but different values of polymeric binder (30vol\% and $50 \mathrm{vol} \%$ ). Stronger polymeric bonds are obvious for the samples with 50vol\% PVA.

The wettability of PVA, in contact with the CPP particles, is a critical factor which has a great influence on the part's green strength. The wettability of a liquid is defined as the contact angle between the droplets on a horizontal surface. A liquid is deemed to be wetting, when the angle varies between 90 and 180 degrees. To achieve an improved performance in the SLS process, the surface tension of the liquid phase must be lower than that of the solid phase. It might be possible by choosing a suitable binary material system.

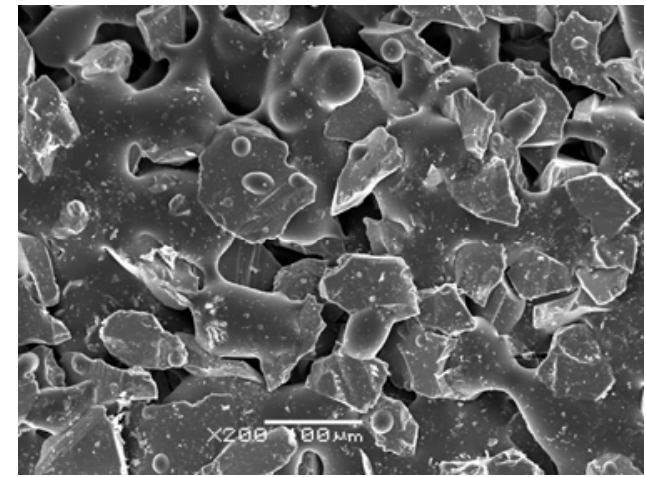

(a)

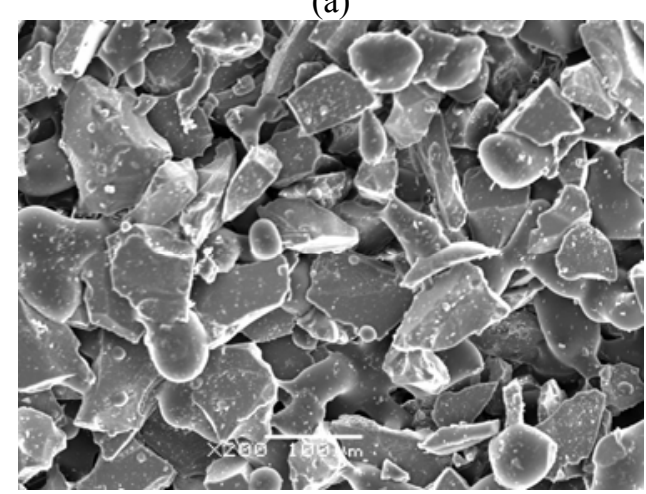

(b)

Fig. 14 SEM of laser sintered samples with (a) 50vol\% PVA\#1 and (b) 30vol\% PVA\#1

Noticing the aforementioned phenomena, as viewed in Fig. 14, PVA may not be a proper choice for liquid phase sintering of CPP, because it does not demonstrate any wellwetted connections. However, the sample in Fig. 15 expresses an inverse behaviour of PVA and CPP, even though it was fabricated with the same laser power and scanning speed $(16 \mathrm{~W}$ and $45 \mathrm{~mm} / \mathrm{s})$. Ceramic particles are suitably wetted and covered with PVA, although only 20 vol\% PVA was used. The arrows evident the PVA bridges between CPP particles. The difference between the samples of Fig. 14 and Fig. 15 is the PVA's molecular weight (MW) and the degree of hydrolysation as listed in Table 2. It is concluded that these two properties of PVA might influence the wettability with CPP and should be studied more in future work.

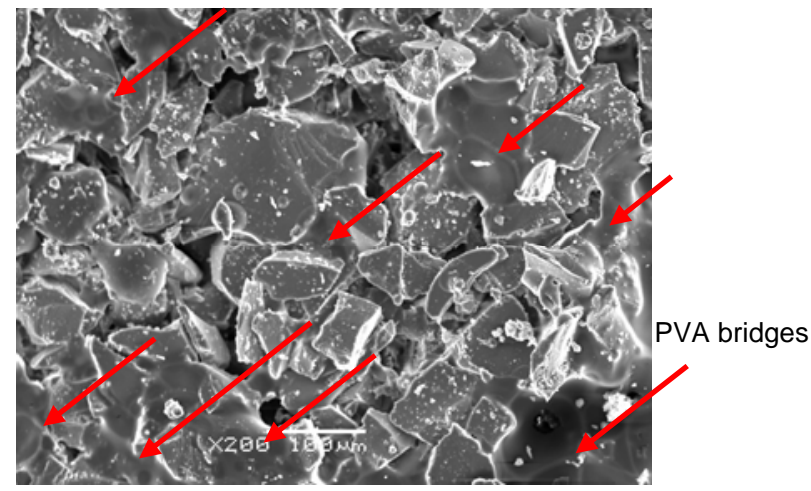

Fig. 15 SEM of laser sintered sample with 20 vol\% PVA \#2 


\section{Conclusion}

The thermal analysis of the selective laser sintering of CPP was conducted through a multi-physics finite element model. Temperature and porosity distributions of the sintered region were investigated versus process parameters. The modeling results suggest that the porosity of the sintered region is a logistic function of the laser scanning speed and a negative logistic function of the laser power. These results can be used in the modification of the process parameters in order to adjust the porosity of the fabricated green parts. In addition, the feasibility study on the SLS of CPP was experimentally performed. Thin single layers of sintered CPP were studied in terms of thickness and microstructure.

\section{Acknowledgments}

The CPP material was produced and provided by the Institute of Biomaterials and Biomedical Engineering at the University of Toronto, under the supervision of Professor Robert M. Pilliar. Their contribution is appreciated by the authors. We are also grateful to Dr. Andrew Pinkerton for his technical advice and the CIHR for providing financial support.

\section{References}

[1] C.K. Chua and K.F. Leong: "Rapid Prototyping: Principles \& Applications in Manufacturing" ed. by C.S. Lim, (World Scientific, 2003) p.173.

[2] R.M. Pilliar, M.J. Filiaggi, J.D. Wells, M.D. Grynpas and R.A. Kandel: Biomaterials, 22, (2001) 963.

[3] J.N. Nelson, N.K. Vail, J.W. Barlow, J.J. Beaman, D.L. Bourel and H.L. Marcus: Ind. Eng. Chem. Res., 34, (1995) 1641.

[4] J.C. Nelson, S. Xue, J.W. Barlow, J.J. Beaman, H.L. Marcus and D.L. Bourel: Ind. Eng. Chem. Res., 32, (1993) 2305.

[5] T.H. Childs, M. Berzins, G.R. Ryder and A. Tontowi: Proceedings of the Institution of Mechanical Engineers, Part B: Journal of Engineering Manufacture, 213(4), (1999) 333.

[6] T.H. Childs and A. Tontowi: Proceedings of the Institution of Mechanical Engineers, Part B: Journal of Engineering Manufacture, 215(4), (2001) 1481.

[7] G.B.M. Cervera and G. Lombera: Rapid Prototyping Journal, 5(1), (1999) 21.

[8] T. Chen and Y. Zhang: Numerical Heat Transfer Part A: Applications, 46(7), (2004) 633.

[9] L. Dong, A. Makradi, S. Ahzi and Y. Remond: Materials Science Forum, 553, (2007) 75.

[10] M.D. Grynpas, R.M. Pilliar, R.A. Kandel, R. Renlund and M. Filiaggi: Biomaterials, 23(9), (2003) 2063.

[11] S.D. Waldman, M.D. Grynpas, R.M. Pilliar and R.A. Kandel: Journal of Biomedical Materials Research, 62(3), (2002) 323.

[12] E. Toyserkani, A. Khajepour and S. Corbin: Optics and Lasers in Engineering, 41(6), (2004) 849.

[13] A. Fathi, E. Toyserkani, A. Khajepour and M. Durali: Journal of Physics D: Applied Physics, 39(12), (2006) 2613.

[14] S. Yagi and D. Kunii: AIChE Journal, 3, (1957) 373.

(Received: June 16, 2008, Accepted: February 20, 2009) 